\section{Birth and adaptive evolution of a hominoid gene that supports high neurotransmitter flux}

\author{
Fabien Burki \& Henrik Kaessmann
}

The enzyme glutamate dehydrogenase (GDH) is important for recycling the chief excitatory neurotransmitter, glutamate, during neurotransmission. Human GDH exists in housekeeping and brain-specific isotypes encoded by the genes GLUD1 and GLUD2, respectively. Here we show that GLUD2 originated by retroposition from GLUD1 in the hominoid ancestor less than 23 million years ago. The amino acid changes responsible for the unique brain-specific properties of the enzyme derived from GLUD2 occurred during a period of positive selection after the duplication event.

Astrocytes are glial cells that surround neurons and have a pivotal role in regulating the flux of glutamate ${ }^{1}$. After neuron firing, this neurotransmitter is inactivated by its uptake into astrocytes, where it is metabolized by several key enzymes ${ }^{1,2}$. One of these enzymes, GDH, catalyzes the oxidative deamination of glutamate to ammonia and $\alpha$-ketoglutarate (generating ATP through the Krebs cycle ${ }^{3}$ ), a reaction that fuels recycling of glutamate by glutamine synthetase ${ }^{2}$. In addition, degradation by GDH seems to be important for the detoxification of glutamate at high concentrations after neuron firing ${ }^{4}$. Altered activity of this enzyme is implicated in human neurodegenerative disorders ${ }^{3}$ and potentially in other neurological deficiencies ${ }^{5,6}$.

In the human brain, GDH exists in two isotypes encoded by GLUD1 and GLUD2 (ref. 4). GLUD1 is an important housekeeping gene expressed in many tissues ${ }^{4}$, whereas GLUD2 is specifically expressed in nerve tissues (brain and retina) and in testis ${ }^{7}$. Unlike GLUD1, the enzyme derived from GLUD2 is highly adapted to functioning in the brain ${ }^{4}$ : it is highly functional in spite of the high GTP levels in the brain; its activity is up to 25 times higher than the basal level, depending on the concentrations of the allosteric activators ADP and L-leucine; and it can function at relatively low $\mathrm{pH}$ levels ${ }^{3,4}$. These properties permit instant activation of the enzyme in the brain under high-frequency firing of the neurons.

GLUD2 is an X-linked intronless gene ${ }^{7}$ that probably originated by retroposition of a spliced mRNA derived from the intron-containing gene GLUD1 on chromosome 10. We could identify only single functional GLUD genes orthologous to human GLUD1 when screening available genome sequences (Supplementary Methods online). Because a primate burst of retroposition, which began $\sim 40-50$ million years $\operatorname{ago}^{8}$, has generated a large number of retrocopies of genes on the primate lineage, we hypothesized that GLUD2 might be a young primate retrogene that originated recently by retroduplication of GLUD1 and then adopted its brain-specific function.

To test this hypothesis, we first gauged the age of GLUD2 by screening for the presence or absence of GLUD2 in primates using PCR with primers flanking the retrogene insertion site. We found that GLUD2 was present only in humans and apes and that the sequence of the insertion site in Old World monkeys (OWMs) reflects the ancestral state, before the GLUD2 insertion (Supplementary Fig. 1 online). Consequently, GLUD2 is a hominoid-specific gene that emerged by retroduplication after the OWM-hominoid split, $\sim 23$ million years ago $^{9}$, but before the separation of the gibbon lineage from that of humans and great apes, $\sim 18$ million years ago 9 .

To trace the evolutionary history of GLUD2, we first sequenced its coding region in the apes and then reconstructed a phylogenetic tree using these sequences along with GLUD1 sequences from humans, an OWM, mice and rats (Fig. 1). An analysis of the substitutional pattern for each branch of the tree (Supplementary Methods online) identified several synonymous substitutions that occurred after the separation of the OWM GLUD1 lineage (node A) but before the duplication event (node B). This suggests that GLUD2 emerged some time after the OWM-hominoid split, substantially less than 23 million years ago. The analysis also identified an excess of nonsynonymous $\left(K_{\mathrm{A}}\right)$ over synonymous substitutions $\left(K_{\mathrm{S}}\right)$ per site $\left(K_{\mathrm{A}} / K_{\mathrm{S}}>1\right)$ for the branches after the duplication event leading to humans and the great apes, indicative of accelerated protein evolution driven by positive selection $^{10}$; this excess was not statistically significant. But a maximum likelihood analysis that tests for selection at certain sites on these branches (Supplementary Methods online) identified a subset of sites with $K_{\mathrm{A}} / K_{\mathrm{S}}$ values significantly greater than 1 (branch B-C, $K_{\mathrm{A}} / K_{\mathrm{S}} \approx$ 4; branch $\mathrm{C}-\mathrm{D}, K_{\mathrm{A}} / K_{\mathrm{S}} \approx 16.4 ; P<0.01$; Fig. 1), suggesting that GLUD2 was subjected to positive selection after the duplication event in the human and great ape ancestor.

Unlike the internal branches that have $K_{\mathrm{A}} / K_{\mathrm{S}}$ values $>1$, all peripheral branches have $K_{\mathrm{A}} / K_{\mathrm{S}}$ values $<1$, indicative of the action of purifying selection (the difference between the internal and external branches is significant, $P<0.05$ ). This might indicate that functional adaptation of GLUD2 driven by positive selection progressively occurred on the line leading to humans and great apes, whereas new functional variants were maintained in the individual human and ape lineages by purifying selection.

In sharp contrast to the evolutionary fate of GLUD2, GLUD1 has been conserved after the duplication event, accumulating no amino acid substitutions $\left(K_{\mathrm{A}} / K_{\mathrm{S}}=0, P<0.01\right)$. This suggests that GLUD1 has been under strong purifying selection after gene duplication and has maintained its original housekeeping function ${ }^{3}$.

Previous studies ${ }^{4}$ using site-directed mutagenesis of GLUD1 at sites that differ from the corresponding residues of GLUD2 identified two 
Figure 1 Phylogenetic tree based on hominoid GLUD2 sequences encoding the mature peptide and the human and African green monkey (AGM; representing OWMs) GLUD1 sequences. Mouse and rat GLUD1 orthologs were used as outgroups. Approximate divergence times in millions of years (Mya) are shown (estimates based on ref. 9). Maximum likelihood $K_{A} / K_{S}$ values and the estimated number of nonsynonymous over synonymous substitutions (in parentheses) for each branch are indicated. Internal nodes are of labeled (A-G) and the two branches under positive selection after the duplication event are shown in bold. Amino acid changes that occurred on these two branches are as follows: branch B-C: A3V, E34K, D142E, S174N, R443S, G456A and N498S; branch C-D: V3L, R39Q, K299R, S331T, M370L and R470H. Amino acid changes E34K, D142E, S174N, R443S, G456A, N498S, S331T, M370L and R470H occurred at sites that are inferred to have been potentially under positive selection $(P>0.95)$. Amino acid changes R443S and G456A provide GLUD2 with brain-specific properties ${ }^{4}$; N498S, S331T, M37OL and R470H do not influence functional differences between GLUD1 and GLUD2. Amino acid changes on the other branches are as

을 follows: branch D-E: I166V, G247R and A321V;

branch A-African green monkey: N139S; branch C-gibbon: E8K, T101A and L377V; branch D-orangutan: I239N, L240V, I275V, L375V and Q441R; branch E-gorilla: S66C, K362R, L365Q and E439D; branch F-chimpanzee: I305L and V321I; branch F-human: R299K and M415L; branch A-G: V4A, g R37K, I275V, A276G, P313V and I434V; branch G-rat: A5T, S41N and F246L. Amino acid change M415L does not influence functional differences between GLUD1 and GLUD2 (ref. 4).

key amino acid changes that are essential for the brain-specific activity and allosteric regulation of GLUD2. One of these changes (G456A) renders GDH functional at high GTP concentrations, and the other (R443S) abolishes basal activity of GDH and causes the mutant enzyme to become dependent on ADP for its function. The latter also changes the $\mathrm{pH}$ at which $\mathrm{GDH}$ shows high activity to the value ( $\mathrm{pH}$ 7.0) found in astrocytes under high neuron firing ${ }^{4}$.

We found that all hominoid GLUD2 sequences carry these two crucial substitutions, and our evolutionary analysis confirmed that they occurred in the first few million years after the origin of GLUD2 in the human and ape ancestor (Fig. 1; branch B-C). Thus, two amino acid substitutions, which emerged during a period of positive darwinian selection, equipped the GLUD2 enzyme with the main properties that allow it to be activated in astrocytes under conditions of intense glutamatergic transmission ${ }^{4}$. Notably, the two sites where these amino acid substitutions occurred are also identified as potentially being positively selected (Fig. 1). But these two substitutions do not explain all the functional differences between the GLUD1 and GLUD2 isoenzymes ${ }^{4}$. For example, GLUD2 requires lower ADP levels for activation than the GLUD1 mutants and is amenable to activation by L-leucine in the absence of ADP.

To identify amino acid changes that may account for these properties of GLUD2, we scrutinized the remaining 16 substitutions in the mature enzyme that occurred after the duplication event leading to the extant human GLUD2 sequence (Fig. 1). Five of these changes do not affect GDH function ${ }^{4}$. In addition, a recurrent mutation (K299R on branch C-D and R299K on branch F-human) probably has no affect on GDH function, as it reinstates the ancestral amino acid (also found in GLUD1). All or several of the remaining nine substitutions in the human and great ape ancestor may have endowed GLUD2 with more refined neural properties (Fig. 1). Six of these changes occurred during the period of positive selection after the duplication event and therefore may be particularly relevant for the functional adaptation of GLUD2.

The origin and adaptive phase of GLUD2 approximately coincide with a period of increased structural and functional complexity, as well as an increase in brain size, in the human and great ape ancestor $^{11}$. The genes ASPM and $M C P H 1$, determinants of brain size, also show accelerated evolution during this time period ${ }^{12,13}$. Large-scale gene expression studies showed that an overall upregulation of genes involved in synaptic transmission and metabolic processes that support it has occurred on the human lineage (e.g., ref. 14), suggesting that higher neuronal activity may have coevolved with greater brain size and other changes.

Consequently, GLUD2 probably contributed to enhanced brain function in humans and apes by permitting higher neurotransmitter flux. Decreased activity of this retrogene in neurodegenerative disorders $^{3}$ suggests that it is probably crucial for sustaining proper glutamate flux in the human brain. Moreover, the additional GLUD2 gene in hominoids might be potentially relevant to enhanced memory-related abilities in humans and apes, as the glutamate dehydrogenase gene was among two candidates identified in a systematic screen for genes upregulated during late memory formation in rats ${ }^{15}$. Thus, GLUD2 may have been important for the evolution of increased cognitive capacities in hominoids.

GenBank accession numbers. GLUD2 coding sequences: chimpanzee, AY588274; gorilla, AY588267; orangutan, AY588268; gibbon, AY588269. GLUD2 insertion site: African green monkey, AY588270. GLUD1 coding sequence: African green monkey, AY677079.

Note: Supplementary information is available on the Nature Genetics website. 


\section{ACKNOWLEDGMENTS}

We thank I. Dupanloup and A. Marques for constructive discussions; E. Farmer,

L. Keller, I. Sanders and W. Wahli for comments on the manuscript; and

A. Reymond for comments on the manuscript and samples. This research was supported by funds available to H.K. from the Center for Integrative Genomics, University of Lausanne, and the Swiss National Science Foundation.

\section{COMPETING INTERESTS STATEMENT}

The authors declare that they have no competing financial interests.

Received 12 April; accepted 19 August 2004

1. Hertz, L., Dringen, R., Schousboe, A. \& Robinson, S.R. J. Neurosci. Res. 57, 417-428 (1999).

2. Sonnewald, U., Westergaard, N. \& Schousboe, A. Glia 21, 56-63 (1997).
3. Plaitakis, A., Metaxari, M. \& Shashidharan, P. J. Neurochem. 75, 1862-1869 (2000).

4. Plaitakis, A., Spanaki, C., Mastorodemos, V. \& Zaganas, I. Neurochem. Int. 43, 401410 (2003).

5. Burbaeva, G. et al. Prog. Neuropsychopharmacol. Biol. Psychiatry 27, 675-680 (2003).

6. Wan, M. \& Francke, U. Am. J. Med. Genet. 78, 169-172 (1998).

7. Shashidharan, P. et al. J. Biol. Chem. 269, 16971-16976 (1994).

8. Zhang, Z., Carriero, N. \& Gerstein, M. Trends Genet. 20, 62-67 (2004).

9. Goodman, M. et al. Mol. Phylogenet. Evol. 9, 585-598 (1998).

10. Li, W.H. Molecular Evolution: Roles of Mutation and Selection in Molecular Evolution (Sinauer Associates, Sunderland, Massachusetts, 1997).

11. Noback, C.R. \& Montagna, W. The Primate Brain: The Evolution of Mental Genes (Meredith Corporation, New York, 1970).

12. Evans, P.D. et al. Hum. Mol. Genet. 13, 489-494 (2004).

13. Evans, P.D., Anderson, J.R., Vallender, E.J., Choi, S.S. \& Lahn, B.T. Hum. Mol. Genet. 13, 1139-1145 (2004).

14. Caceres, M. et al. Proc. Natl. Acad. Sci. USA 100, 13030-13035 (2003).

15. Cavallaro, S. et al. Proc. Natl. Acad. Sci. USA 94, 9669-9673 (1997). 